Fernando Martínez ORCID 0000-0002-6916-4738 Neurocirujano. Profesor Agregado de Neurocirugía

Rodrigo Moragues ORCID: 0000-0002-3739-183X Neurocirujano. Profesor Adjunto de Neurocirugía.

Gonzalo Bertullo ORCID: 0000-0002-3171-7915 Neurocirujano. Asistente de Neurocirugía.

Federico Salle ORCID 0000-0002-8604-1502 Neurocirujano. Asistente de Neurocirugía.

Gastón Duarte ORCID: 0000-0002-1559-7677 Anestesista. Asistente de Anestesiología

Ronnie Henderson ORCID: 0000-0001-5750-6676

Anestesista. Asistente de Anestesiología.

Fabrizzio Sacchet ORCID: 0000-0002-4628-7508

Anestesista. Asistente de Anestesiología.

Federico Preve ORCID: 0000-0002-3800-0159 Neurólogo. Asistente de Neurología.

Andrés Lescano ORCID 0000-0002-5443-4547 Neurólogo. Neuropsicólogo

Gustavo Grünberg ORCID: 0000-0001-7687-8141 Anestesista. Ex Profesor Adjunto de Anestesiología.

Lorena Tambasco ORCID: 0000-0003-3258-527X Anestesista.

Paola Alcarraz ORCID: 0000-0002-5224-6163

Anestesista. Asistente de Anestesiología

Elena Vignoli ORCID: 0000-0003-2309-599x Anestesista.

\section{Cirugía con despertar operatorio para procesos expansivos cerebrales. Reporte de 20 casos.}

\author{
Surgery with operative awakening for expansive brain processes. Report \\ of 20 cases. \\ Cirurgia com despertar operativo para processos cerebrais expansivos. \\ Relato de 20 casos.
}

Resumen: Introducción. La cirugía de lesiones expansivas cerebrales con paciente despierto es una técnica que se usa de forma cada vez más frecuente. Esto se debe a que es una técnica costoefectiva para realizar resección de tumores cerebrales de forma amplia y segura. Resultado. Se presentan 20 pacientes operados con dicha técnica. Se trató de 13 hombres y 7 mujeres, rango etario 16 - 67 años, portadores de 17 lesiones tumorales y 3 lesiones vasculares (angiomas cavernosos). Se realizaron 22 cirugías ya que dos pacientes se intervinieron 2 veces. Se logró resección supra-máxima en 3 casos, completa en 9, subtotal en 5 y parcial en 2 pacientes. Un paciente no se pudo operar debido a que presentó un despertar inadecuado y el procedimiento se suspendió. En cuanto a las complicaciones, $18 \%$ de los pacientes presentaron crisis intraoperatorias, pero las mismas no impidieron el desarrolló del procedimiento con normalidad luego de yugulada la crisis. Dos pacientes ( $9 \%$ de los procedimientos) presentaron un despertar inadecuado. En un caso la lesión se resecó completamente de todas formas, en el otro se suspendió el procedimiento. $18 \%$ de los pacientes presentaron una peoría funcional transitoria y un $4.5 \%$ presentó una peoría definitiva (paresia severa). Un solo paciente (4.5\%) presentó una infección del colgajo que requirió retiro de la plaqueta ósea y colocación de una placa de acrílico en diferido. Conclusiones. Las cifras presentadas por los autores están en concordancia con las de los centros regionales e internacionales de referencia.

Palabras clave: Glioma cerebral, metástasis, cirugía con despertar operatorio, monitoreo neurofisiológico.

Abstract: Introduction. Expansive brain injury surgery with awake patients is a technique that is being used more and more frequently. This is because it is a cost-effective technique for performing brain tumor resection widely and safely. Outcome. Twenty patients operated with this technique are presented. There were 13 men and 7 women, age range 16 - 67 years, carriers of 17 tumor lesions and 3 vascular lesions (cavernous angiomas). 22 surgeries were performed since two patients underwent surgery twice. Supra-maximal resection was achieved in 3 cases, complete in 9 , subtotal in 5 , and partial in 2 patients. One patient could not be operated on due to inadequate awakening and the procedure was suspended. Regarding complications, $18 \%$ of the patients presented intra-operative seizures, but they did not prevent the normal development of the procedure after the crisis was jugulated. Two patients ( $9 \%$ of the procedures) had inadequate awakening. In one case the lesion was completely resected anyway, in the other the procedure was suspended. $18 \%$ of the patients presented a transitory functional deterioration and $4.5 \%$ presented a definitive worsening (severe paresis). Only one patient (4.5\%) had a flap infection that required removal of the bone plate and placement of a delayed acrylic plate. Conclusions. The figures presented by the authors are in accordance with those of the regional and international reference centers.

Key words: Cerebral glioma, metastasis, surgery with operative awakening, neurophysiological monitoring. 
Resumo: Introdução. A cirurgia de lesão cerebral extensiva com pacientes acordados é uma técnica cada vez mais utilizada. Isso ocorre porque é uma técnica econômica para realizar a ressecção de tumor cerebral de forma ampla e segura. Resultado. São apresentados 20 pacientes operados com essa técnica. Eram 13 homens e 7 mulheres, com idades entre 16 e 67 anos, portadores de 17 lesões tumorais e 3 lesões vasculares (angiomas cavernosos). Foram realizadas 22 cirurgias, pois dois pacientes foram operados duas vezes. A ressecção supramáxima foi alcançada em 3 casos, completa em 9, subtotal em 5 e parcial em 2 pacientes. Um paciente não pôde ser operado devido ao despertar inadequado e o procedimento foi suspenso. Em relação às complicações, $18 \%$ dos pacientes apresentaram convulsões no intra-operatório, mas não impediram o desenvolvimento normal do procedimento após a jugulação da crise. Dois pacientes (9\% dos procedimentos) tiveram despertar inadequado. Em um caso a lesão foi totalmente ressecada de qualquer maneira, no outro o procedimento foi suspenso. $18 \%$ dos pacientes apresentaram uma deterioração funcional transitória e 4,5\% apresentaram piora definitiva (paresia grave). Apenas um paciente (4,5\%) apresentou infecção do retalho que exigiu a retirada da placa óssea e colocação de placa acrílica retardada. Conclusões. Os números apresentados pelos autores estão de acordo com os dos centros de referência regionais e internacionais.

Palavras-chave: Glioma cerebral, metástase, cirurgia com despertar operatório, monitoramento neurofisiológico.

Recibido: 18/04/2021 - Aceptado: 04/09/2021

Instituto de Neurología. Servicio de Neurocirugía. Cátedra de Anestesiología. Hospital de Clínicas - "Dr. Manuel Quintela" - Facultad de Medicina, Universidad de la República. Sanatorio Americano. Mutualista Cooperativa Médica de Canelones (COMECA). Uruguay.

Correspondencia. E-mail: fermartneuro@gmail.com 


\section{Introducción}

Las técnicas de monitoreo de funciones neurológicas son conocidas desde hace más de 80 años a partir de los trabajos de Penfield, pero han tenido un gran desarrollo en los últimos 30 años ${ }^{(1)}$.

Hay diversas formas de monitorizar funciones neurológicas en procedimientos quirúrgicos: uso de neuro-estimulación en cirugía de nervios periféricos, monitoreo de función de vías largas motoras y sensitivas en cirugía de tumores medulares, testeo de función de pares craneanos en cirugía de tumores de fosa posterior, neuro-estimulación cortical o de vías de sustancia blanca para tumores cerebrales bajo anestesia general, monitoreo de funciones motoras, sensitivas, visuales y cognitivas con paciente despierto para patologías tumorales, vasculares y trastornos del movimiento ${ }^{(2-10)}$.

El objetivo de estas técnicas es detectar de forma precoz las alteraciones funcionales de áreas corticales, vías de sustancia blanca y nervios periféricos, para prevenir la lesión estructural y por tanto, aumentar la chance de preservación de la función ${ }^{(11-15)}$.

La cirugía con despertar operatorio para lesiones cerebrales es una técnica que se usa desde hace décadas en el contexto del tratamiento de trastornos funcionales (enfermedad de Parkinson, por ejemplo), epilepsia, lesiones vasculares o tumores ${ }^{(2,16)}$.

Desde los años '80 ha habido un auge progresivo de la cirugía de tumores con despertar operatorio como una herramienta que permite hacer resecciones de lesiones con un riesgo de pérdida funcional menor. Esto es especialmente importante en algunos tipos de lesiones, como los gliomas de bajo grado, que afectan a individuos jóvenes y que tienen un pronóstico vital relativamente bueno a 5 años. En este grupo de pacientes es fundamental lograr no solo tiempo de sobrevida, sino calidad de la misma ${ }^{(15,17,18)}$.

Por estas razones, el paradigma de la cirugía con despertar operatorio es lograr la máxima resección lesional con mínimo riesgo funcional. Esto es lo que Duffau ha definido como neurocirugía oncológica funcional, cuyo precepto es resecar las lesiones de forma muy amplia (incluso tejido que parece "normal"), guiándose por la preservación de la función (11-13, 17, 19-26).

Los autores presentan su experiencia con una serie pacientes operados por lesiones expansivas tumorales o vasculares con la técnica de despertar operatorio en nuestro medio.

\section{Metodología}

Se realizó una revisión retrospectiva de historias clínicas de pacientes operados mediante técnica de despertar operatorio por un mismo grupo de cirujanos entre julio de 2014 y agosto de 2018. Uno de los autores (FM) estuvo presente en todas las cirugías como operador principal o primer ayudante. Los pacientes se operaron en diferentes centros y con diferentes grupos de anestesistas.

Se recogieron los siguientes datos: ficha patronímica, tipo de lesión (vascular o tumoral), ubicación en el cerebro, tipo de monitoreo realizado, grado de resección de la lesión, conservación de la función testada, y aparición de complicaciones vinculadas al monitoreo o la cirugía.

Los datos se tabularon y analizaron con el programa Excel (Office 365, Microsoft Corporation, Redmont, Washington, EEUU).

\section{a) Procedimiento de selección de pacientes}

El paciente tiene una primera entrevista con el cirujano, quien decide si es aplicable el despertar operatorio ${ }^{(27)}$. Esto se basa en características de la lesión: extensión, relación con áreas de corteza o sustancia blanca vinculados a funciones elocuentes (cognición, motricidad, lenguaje, visión, atención visuoespacial, sensibilidad, área visual, etc).

Si la lesión cumple con alguna de estas características, se plantea la posibilidad de despertar operatorio, dando amplia información del procedimiento y los testeos. Se dejan un intervalo de tiempo para que se procese la información.

En una segunda entrevista se le vuelve a explicar el procedimiento y se le da la oportunidad de hacer preguntas nuevamente. Posteriormente el paciente es evaluado antes de la cirugía por el neurólogo que hará el testeo intra-operatorio y por el anestesista. En la evaluación preanestésica se presta atención en explicar detalladamente el procedimiento a realizar para 
obtener la máxima colaboración del paciente, la cual es imprescindible para lograr el éxito del procedimiento.

Se discute dentro del equipo y se decide de común acuerdo la realización del procedimiento.

\section{b) Procedimiento anestésico}

Previo a comenzar la cirugía se prueba la tolerancia a la posición quirúrgica con el paciente despierto para asegurar su confort (Figura 1).

Figura 1: Disposición general de la sala de neurocirugía para un procedimiento con despertar operatorio. La posición quirúrgica

usada actualmente es el decúbito lateral independientemente de la ubicación de la lesión.

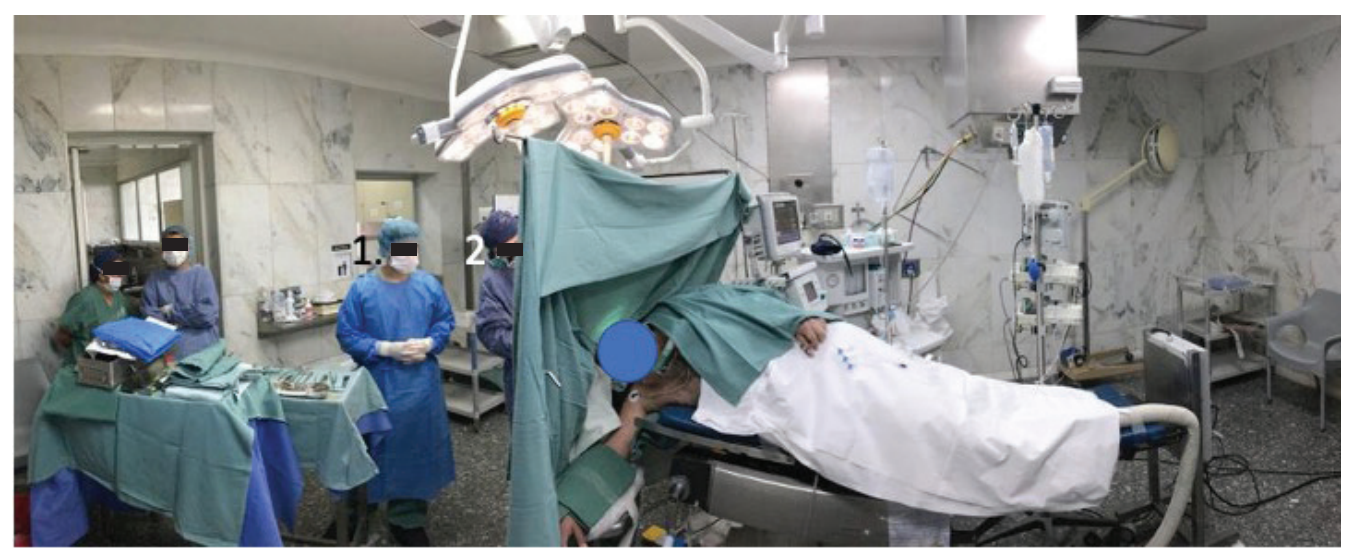

El procedimiento inicia con la colocación de máscara laríngea bajo anestesia general.

Se realiza anestesia total intravenosa por en base a Propofol, Remifentanil y Dexmedetomidina. Se adecúan las dosis de Propofol y Remifentanyl para lograr una medición de Entropía (SE) de 50 (concentraciones en sitio efecto de $1,4 \mathrm{ug} / \mathrm{ml}$ y $1,8 \mathrm{ng} / \mathrm{ml}$ promedio respectivamente). La Dexmedetomidina se administra en dosis carga de $1 \mathrm{ug} / \mathrm{kg}$ en 15 minutos y luego una infusión de $0,4 \mathrm{ug} / \mathrm{kg} / \mathrm{h}$.

La vía aérea se aborda con máscara laríngea Supreme 4 en hombres y 3 en mujeres (LMA Supreme Airway Management, Athlone, Irlanda), sin uso de relajantes musculares ${ }^{(28)}$.

Se administra dexametasona $8 \mathrm{mg}$ y ondansetrón $8 \mathrm{mg}$ i/v inmediatamente a la inducción anestésica como profilaxis para náuseas y vómitos, y antibioticoterapia profiláctica con 2 grs de Cefazolina.

Se coloca cabezal tipo Mayfield (Doro, Black Forest Medical Group, Freiburg, Alemania) haciendo previamente bloqueo anestésico con lidocaína y bupivacaína de los puntos supraorbitario, temporal superficial, auricular posterior y occipital del lado a operar más el supraorbitario contralateral. Se bloquean también los puntos donde se colocará el cabezal (Figuras 2 y 3).

Figura 2: Bloqueo de los nervios del cuero cabelludo.

A: Nervio supraorbitario B: Nervio auriculotemporal C: Nervio auricular D: Nervio Occipital.

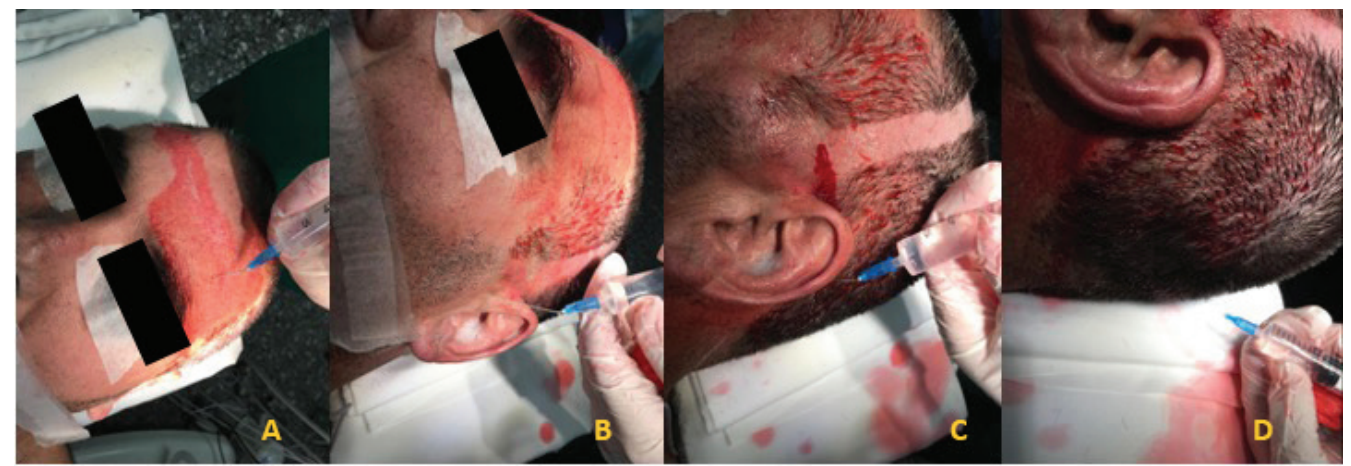



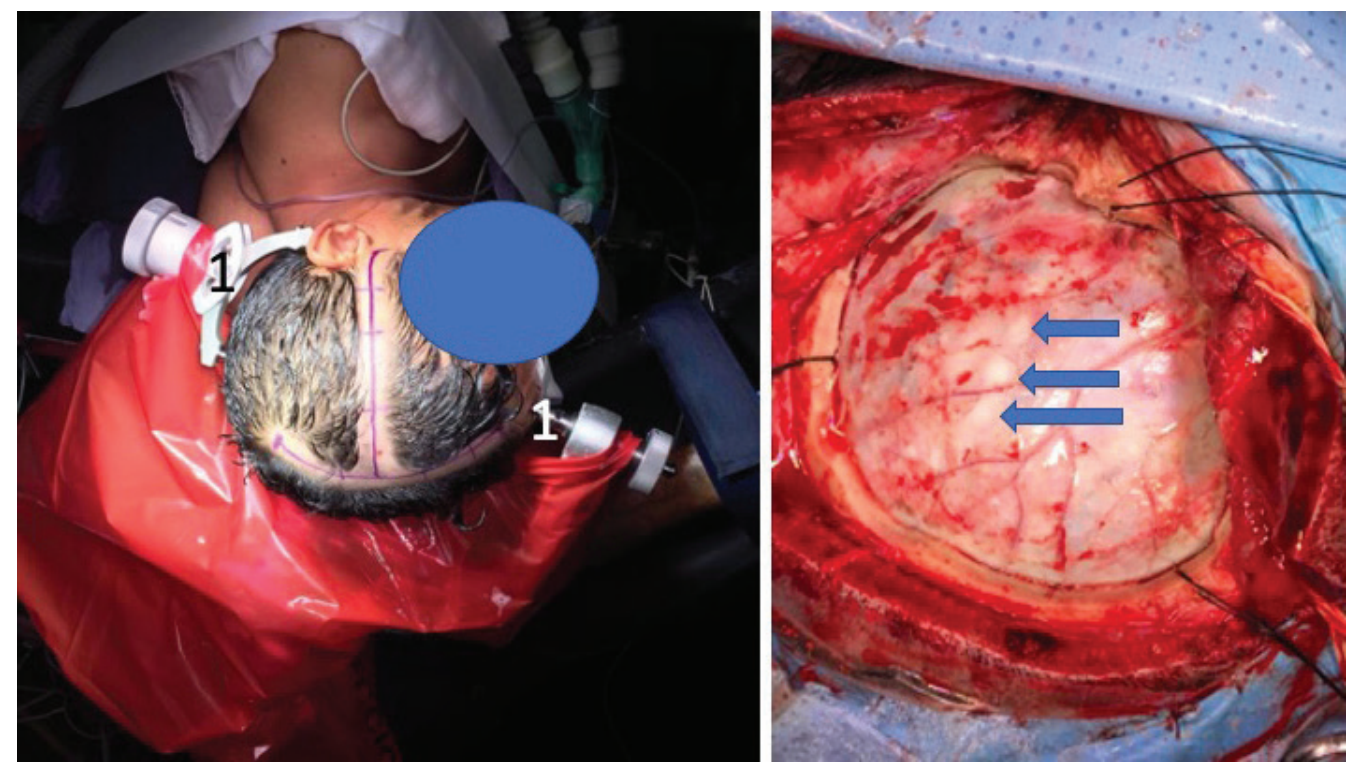

Se hace el abordaje en piel, la craneotomía y anestesia de la duramadre (Figura 3). La misma se abre luego de tener despierto al paciente para evitar que el cerebro protruya a través de la apertura dural durante el despertar.

Una vez completado el abordaje se suspenden las infusiones de Propofol y Remifentanyl y se reduce la Dexmedetomidina a $0.1-0.2 \mathrm{ug} / \mathrm{kg} / \mathrm{h}$. Al despertar se retira la máscara laríngea y se coloca un catéter nasal con oxígeno a $3 \mathrm{l} / \mathrm{min}$. Con el paciente despierto se le pregunta si hay algún sitio de molestia o dolor y se refuerza la anestesia local, así como se corrigen posibles posiciones incómodas.

Una vez concluida la evaluación neurológica intra-operatoria y la resección de la lesión, se hace pre-oxigenación con oxígeno al $100 \%$, se reanudan las infusiones de Propofol y Remifentanil, y se duplica la dosis de Dexmedetomidina. Se recoloca la máscara laríngea, sin el uso de relajantes musculares y se realiza el cierre.

Se hace traslado a la Sala de Recuperación Post Anestésica (SRPA) con el paciente despierto, ventilando espontáneamente con máscara de flujo libre, monitorizado.

En todos los casos el postoperatorio cursó las primeras 24-48 horas en Centro de Tratamiento Intensivo (CTI).

\section{c) Estimulación y testeos intra-operatorios}

Las funciones a testear son motricidad, lenguaje y cognición, aunque dependiendo del paciente y del área a operar se pueden testear otras funciones (reconocimiento de caras, vía visual).

Para testear función motora, se pide al paciente que mueva el hemicuerpo contralateral al lado operado. Si la cirugía es en la convexidad cerebral se testea el movimiento del miembro superior y la cara, en tanto si es en cara interna, se testea la motilidad del miembro inferior.

El lenguaje se testea usando el test de denominación de Boston ${ }^{299}$. El paciente va reconociendo y nombrando en voz alta cada una de las figuras que se le muestran mientras se hace estimulación de la corteza cerebral.

Se realiza siempre un testeo previo a la cirugía para evaluar las imágenes en las cuales hay fallos para estar seguros de que un fallo intra-operatorio no ocurre porque el paciente no conoce la imagen o porque tiene un déficit previo.

Por ejemplo, en el protocolo hay imágenes de un castor, un camello y un uniocornio. Dependiendo del nivel educativo del paciente, puede reconocer al castor como un carpincho o una nutria. En condiciones normales se debería interpretar esto como una parafasia semántica. Si no nomina al animal por no conocerlo, se puede interpretar como una anomia. Por estos motivos es que es absolutamente necesario el testeo previo. 
Para cognición se usa el test de pirámides y palmeras o PPTT ${ }^{(30)}$. Se pide al paciente que asocie entre si dos de tres imágenes (por ejemplo, un astronauta, un cohete y una casa, se le solicita que reconozca cada figura pero además que diga cuales se relacionan entre sí de forma más adecuada).

Mientras se hace el testeo correspondiente, se estimula la corteza cerebral o fascículos de sustancia blanca y si el paciente tiene un fallo (interrumpe el movimiento o la nominación de objetos), esa área puntual está en relación con la función y debe preservarse.

Los parámetros de estimulación utilizados fueron los siguientes: estimulación con electrodo bipolar, fuente de energía BEIC (Rosario, Argentina), pulso de tipo bifásico, amplitud de onda $60 \mathrm{~Hz}$, duración del pulso $1 \mathrm{~ms}$, duración del estímulo 4 segundos. La intensidad del estímulo comenzó en todos los casos en $1,5 \mathrm{~mA}$ o $2,5 \mathrm{~mA}$ y se fue subiendo de a $0.5 \mathrm{~mA}$ hasta obtener respuesta o hasta llegar a $5 \mathrm{~mA}$ como valor máximo. Los estímulos se realizan en toda el área cortical expuesta antes de la resección y durante la misma. La estimulación de sustancia blanca se hace a medida que avanza la cirugía, con los mismos parámetros.

Consideramos respuesta positiva cuando la función testada sufre una alteración: detención del movimiento o sensación de dificultad al realizar el mismo, detención del lenguaje (speech arrest), anomias, repetición o perseverancia verbal, parafasias fonémicas o semánticas, alucinaciones visuales, falta de reconocimiento de las imágenes mostradas, dificultad para conectar entre si las imágenes del protocolo PPTT.

Terminada la cirugía, se hace una valoración del lenguaje y motricidad en el postoperatorio, así como en los controles posteriores ambulatorios.

\section{Resultados}

Se presentan 20 pacientes en los que se realizaron 22 cirugías. Se trató de 7 mujeres y 13 hombres, rango etario 16 a 67 años, que presentaron 17 lesiones tumorales y 3 lesiones vasculares.

Los datos sobre topografía de las lesiones, tipo de testeo y grado de resección se muestran en la tabla 1.

Tabla 1: Lesiones operadas mediante despertar operatorio $(\mathrm{N}=20)$. Referencia GBG: Glioma de bajo grado, GBM: Glioblastoma heteromorfo, izq: izquierdo, Rec Caras: reconocimiento de caras, FTI: Frontotemporoinsular

\begin{tabular}{|l|l|l|l|l|}
\hline \multicolumn{1}{|c|}{ Tipo de Lesion } & \multicolumn{1}{|c|}{ Ubicación } & \multicolumn{1}{|c|}{ Tipo de monitoreo } & $\begin{array}{c}\text { Conservación de } \\
\text { función }\end{array}$ & \multicolumn{1}{|c|}{$\begin{array}{c}\text { Grado de } \\
\text { resección }\end{array}$} \\
\hline Cavernoma & Temporal izq & Lenguaje & Peoría transitoria & Completa \\
\hline Glioma GIII & Premotor derecho & Motor & Peoría transitoria & Completa \\
\hline GBH & Temporal izq & Lenguaje & Conservado & Subtotal \\
\hline GBG & Frontal derecho & Motor, praxias & Conservado & Supramáximo \\
\hline Metástasis & Carrefour izq & lenguaje/motor & Conservado & Completa \\
\hline GBH & Carrefour izq & lenguaje/motor & Mejoría & Completa \\
\hline Glioma GIII & Temporal izq & lenguaje/motor & Conservado & Subtotal \\
\hline Glioma GIII & FTI izq & lenguaje/motor & Peoría transitoria & Parcial \\
\hline GBG & Frontal derecho & Motor & Conservado & Supramáximo \\
\hline GBG & Frontal derecho & Motor, praxias, gnosias & Conservado & Supramáximo \\
\hline Cavernoma & Frontal izq & Lenguaje & Conservado & Completa \\
\hline Metástasis & Parietal izq & No colaboró & Conservado & Completa \\
\hline GBH & Broca izq & Lenguaje & Conservado & Subtotal \\
\hline GBG & Temporal derecho & Rec caras & Conservado & Completa \\
\hline Cavernoma & Frontal izq & lenguaje/motor & Conservado & Completa \\
\hline GBG & Frontal izq & No & N0 & SUSPENSION \\
\hline GBG & Occipital derecho & Visual motor & Conservado & Subtotal \\
\hline GBG & Frontal derecho & Motor cognitivo & Conservado & Subotal \\
\hline GBG & Frontal izquierdo & Motor lenguaje & Peoria transitoria & Total \\
\hline GBG & FTI izq & Motor lenguaje & Peoria & Parcial \\
\hline
\end{tabular}


Dos pacientes fueron operados en dos oportunidades debido a que presentaban lesiones que no pudieron ser resecadas en una primera cirugía por la extensión lesional. Se trató de extensos gliomas fronto-temporo-insulares izquierdos. Durante la primera resección los pacientes manifestaron cansancio durante el monitoreo y comenzaron a colaborar menos en los testeos, por lo que se decidió detener la cirugía y reprogramarla dado que el resultado de la evaluación neurológica no se consideró fiable.

En la segunda oportunidad se resecó más masa tumoral, pero en ambos quedó lesión infiltrando la ínsula y ganglios basales por lo que se hizo radioterapia (resección parcial, glioma grado III).

Otros dos pacientes con lesiones frontales derechas recibieron resecciones muy amplias pero incompletas, con cirugía bajo anestesia general sin despertar. Se decidió hacer un segundo procedimiento para ampliar el margen con seguridad funcional. En uno de ellos se logró resección supra-maxima y en el otro resección sub total. En este último caso se tuvo confirmación anatomopatológica intraoperatoria de negatividad de los bordes de lesión, pero en la Resonancia Magnética (RM) se identificó una imagen mínima sobre el cuerpo calloso en el hemisferio contralateral. Si bien la misma es dudosa, se consideró como un posible remanente y por ello catalogamos la resección como subtotal.

El quinto paciente presentó una lesión occipital derecha en la que se hizo biopsia por parte de otro equipo quirúrgico. La biopsia mostró que se trataba de un glioma de bajo grado. Ante el riesgo de compromiso de la vía visual, el cirujano tratante decidió no hacer resección de la lesión y se indicó

radioterapia. Se operó con despertar operatorio por parte de nuestro equipo, haciendo monitoreo de la vía visual, logrando una resección subtotal sin compromiso visual.

Dos pacientes fueron operados por otros equipos por gliomas de alto grado. Ambos presentaron recidivas en relación con áreas o vías del lenguaje y nuestro equipo realizó la reintervención de la recidiva con despertar para intentar preservar el lenguaje.

En la Tabla 2 se presentan las complicaciones registradas en el grupo.

Tabla 2: Complicaciones de la cirugía con despertar operatorio (20 pacientes, 22 cirugías).

\begin{tabular}{|l|l|l|}
\hline Complicación & N: 22 cirugias & $\%$ \\
\hline Infección & 1 & 4.5 \\
\hline Despertar inadecuado & 2 & 9 \\
\hline Crisis epilépticas & 4 & 18 \\
\hline Hinchazón & 2 & 9 \\
\hline Peoría transitoria & 4 & 18 \\
\hline Peoría definitiva & 1 & 4.5 \\
\hline
\end{tabular}

Dos pacientes no lograron un nivel de colaboración suficiente para realizar el procedimiento de forma exitosa (despertar inadecuado). Uno de los pacientes presentaba una metástasis del carreofour temporo-parietal, que no alcanzó un nivel de vigilia adecuado. El procedimiento se realizó finalmente bajo anestesia general luego de más de 30 minutos de esperar un nivel adecuado de vigilia. La segunda paciente se despertó excitada, por lo que se sedó. Se intentó en dos oportunidades más el despertar con el mismo resultado. Se decidió no proseguir la cirugía y reprogramarla. En la tomografía computada (TC) de control se evidenció un sangrado intra-tumoral. No queda claro el origen del mismo, visto que nunca se trabajó sobre el tumor. Esta paciente se reprogramó y se operó bajo anestesia general.

Dos pacientes presentaron hinchazón cerebral que se manejó con medidas médicas en el intra-operatorio. Ninguno presentó secuelas relacionadas con este inconveniente intraoperatorio.

Cuatro pacientes presentaron crisis epilépticas motoras o alteraciones de vigilia vinculadas a crisis que se yugularon con suero frío y medicación anticonvulsiva. En estos pacientes se esperó que las crisis se detuvieran y que la conciencia se recuperara adecuadamente. Las crisis no tuvieron más impacto que el prolongar el tiempo operatorio.

Un paciente presentó una infección de la herida operatoria que requirió el retiro de la plaqueta ósea en diferido.

Cinco pacientes presentaron peoría de la función motora o del lenguaje en el postoperatorio inmediato. 4/5 recuperaron la función en el correr de los primeros días o semanas del 
postoperatorio con rehabilitación. 1/5 presentó una peoría definitiva de un déficit del lenguaje, pero puede comunicarse de forma adecuada a pesar de dicho déficit.

No hubo muertes vinculadas a la cirugía en este grupo de pacientes.

Las figuras 4 a 6 muestran casos clínicos representativos de la muestra analizada.

Figura 4 A: Recidiva de glioblastoma frontal izquierdo. Tomografía de cráneo donde se observa a nivel frontal izquierdo una lesión hipercaptante en relación con la topografía del área de Broca y el fascículo longitudinal superior.

Figura 4 B: A izquierda se marca el área de seguridad de resección (flechas) obtenida mediante monitoreo del lenguaje. A derecha el lecho quirúrgico luego de una resección amplia (1).
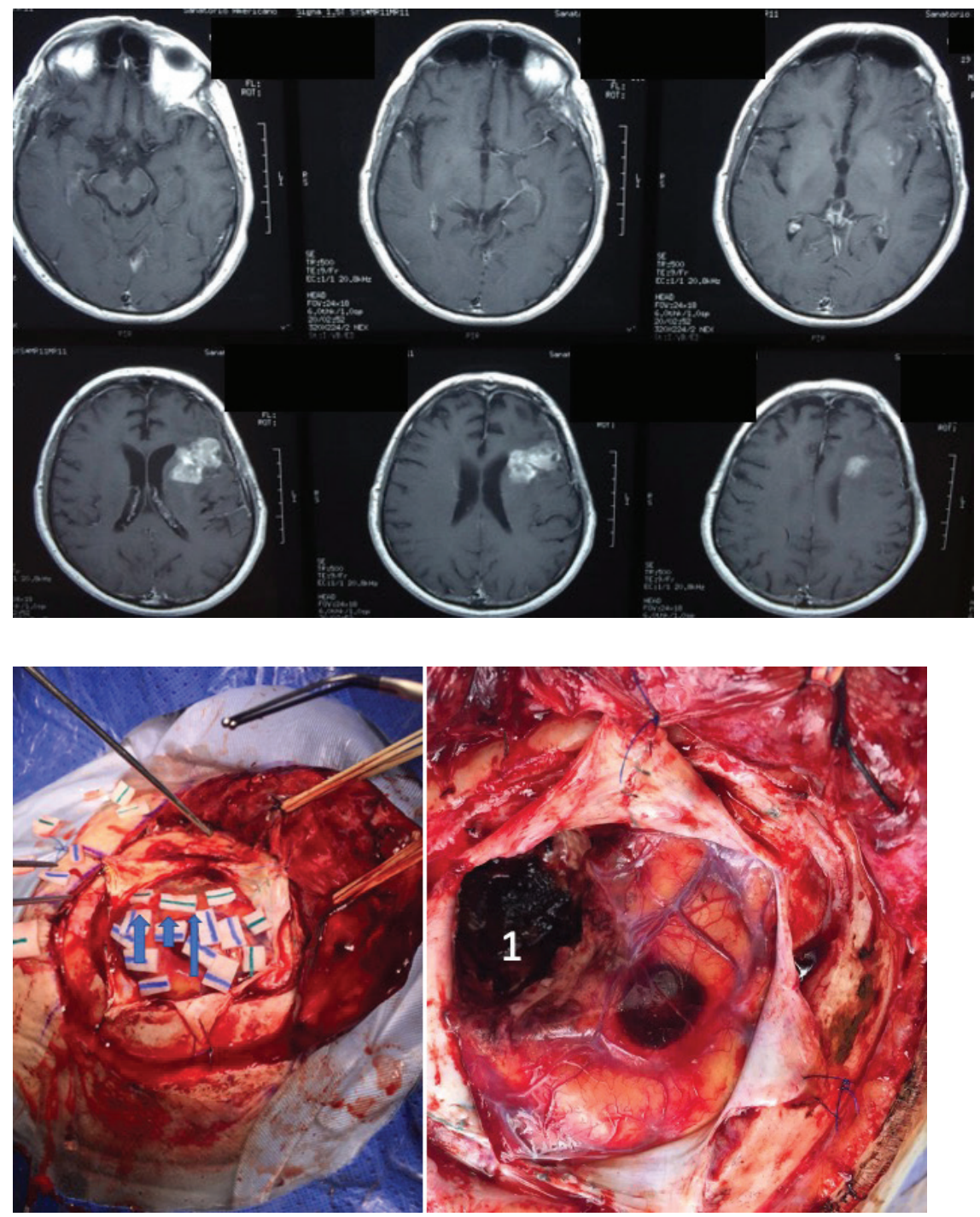

Figura 5 A: Oligodendroglioma grado II. Resonancia Magnética corte axial. Arriba a la izquierda se ve una extensa lesión frontal derecha con importante efecto de masa. Se realizó una primera cirugía con anestesia general, haciendo una resección amplia y posteriormente se planificó una cirugía con despertar para ampliar el margen. Arriba a la derecha se ve el lecho con líquido y material hemostático, abajo el lecho quirúrgico evolucionado con una resección subtotal (mínimo remanente en el cuerpo calloso).

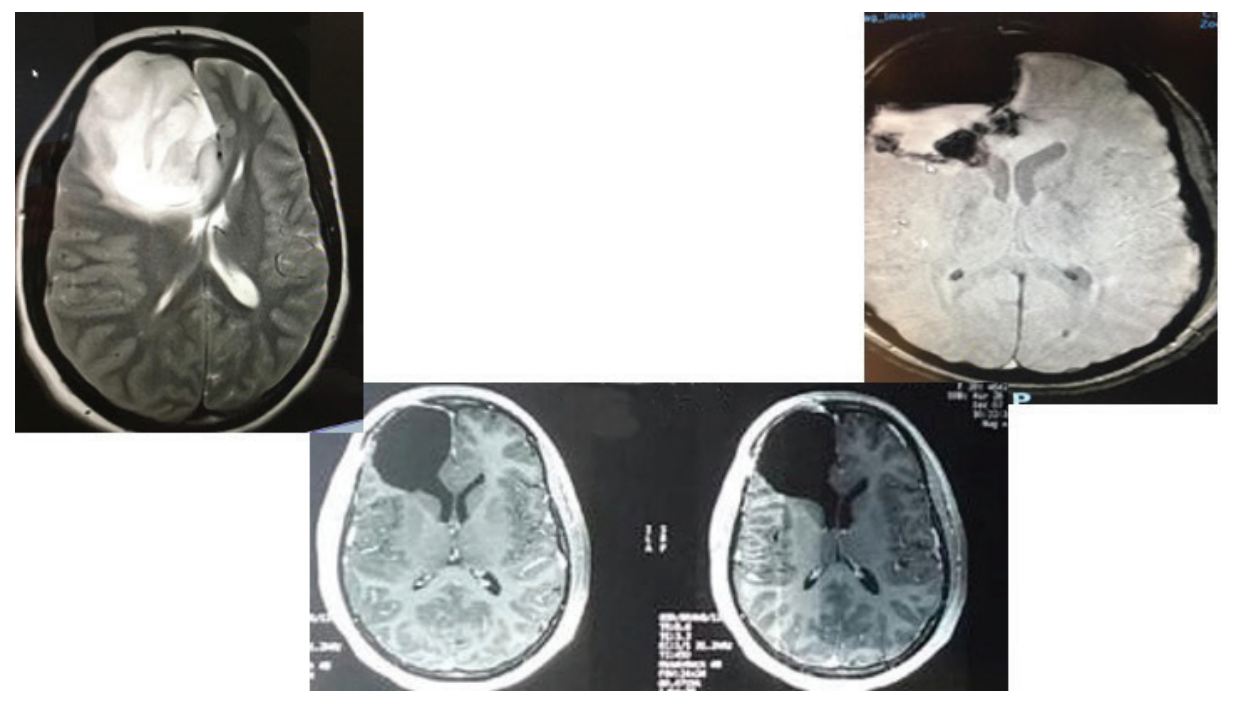


Figura 5 B: Resonancia Magnética corte sagital. Arriba a la izquierda puede verse la voluminosa masa tumoral. Arriba a la derecha, la primer resección amplia, pero incompleta. Abajo, la ampliación de la resección llegando hasta el ventrículo con conservación funcional completa.

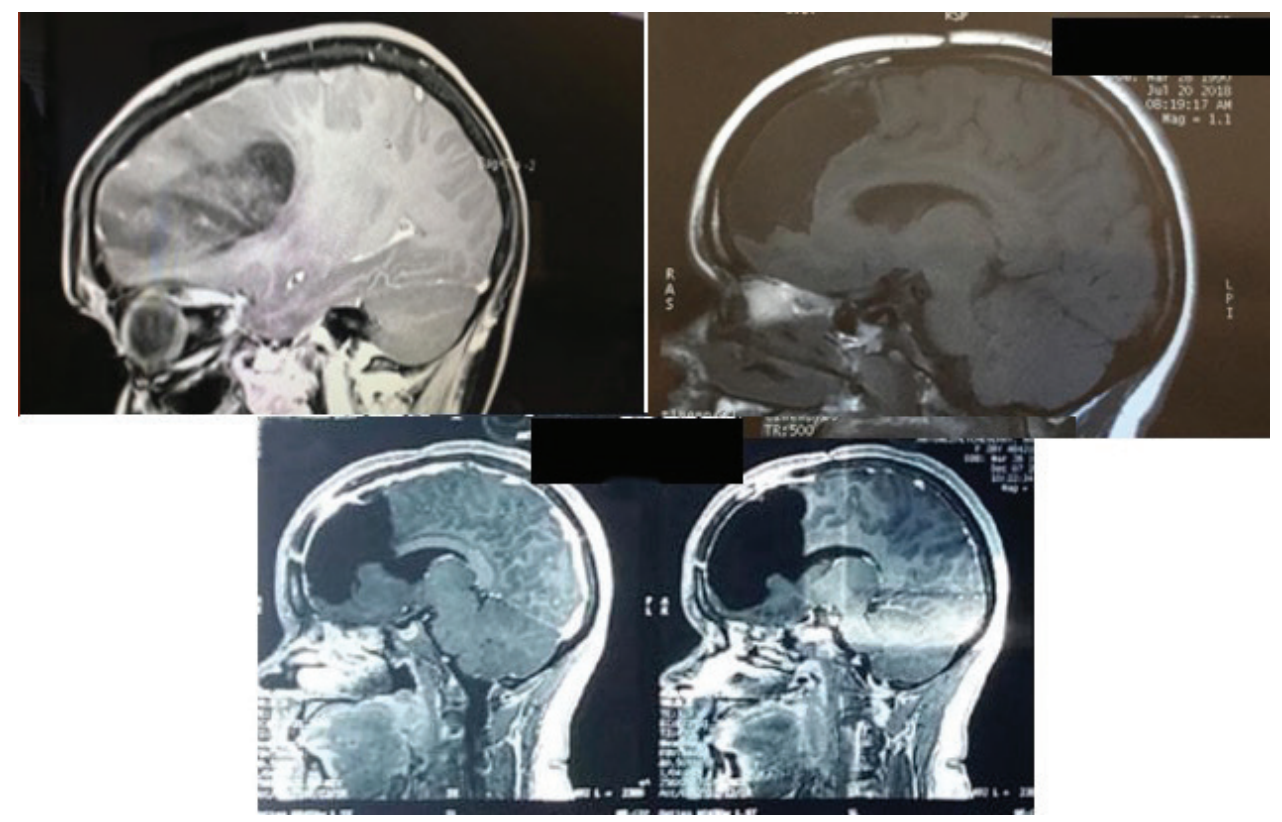

Figura 6 A: Glioma de bajo grado fronto-témporo insular izquierdo. Resonancia Magnética donde se observa la lesión trilobular.

Figura 6 B: A la izquierda, en la primera cirugía se identificó la fisura silviana (4), lóbulo temporal (3) y área del lenguaje (5). A la derecha se observa el abordaje transopercular temporal (flecha voluminosa),

transopercular frontal (7), preservándose cerrada la fisura silviana (flecha pequeña). Los tags marcados muestran zonas de disfunción del lenguaje presentes al estímulo.
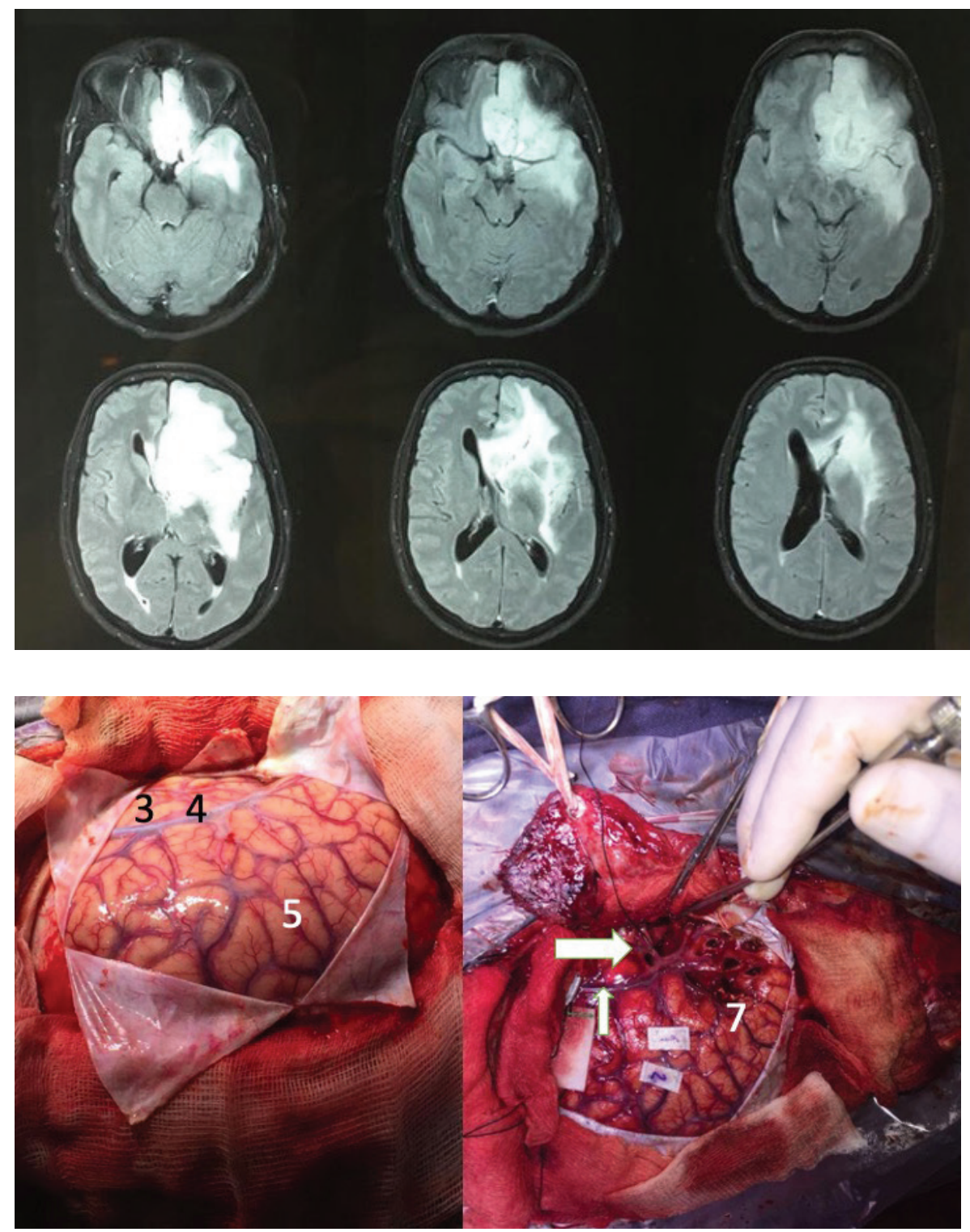
Figura 6 C: Resonancia Magnética en diferentes secuencias y planos donde se muestra la amplia cavidad de resección (estrella). En esta lesión fueron necesarias

dos cirugías, la resección completa no es posible con conservación funcional dada la extensión de la misma.

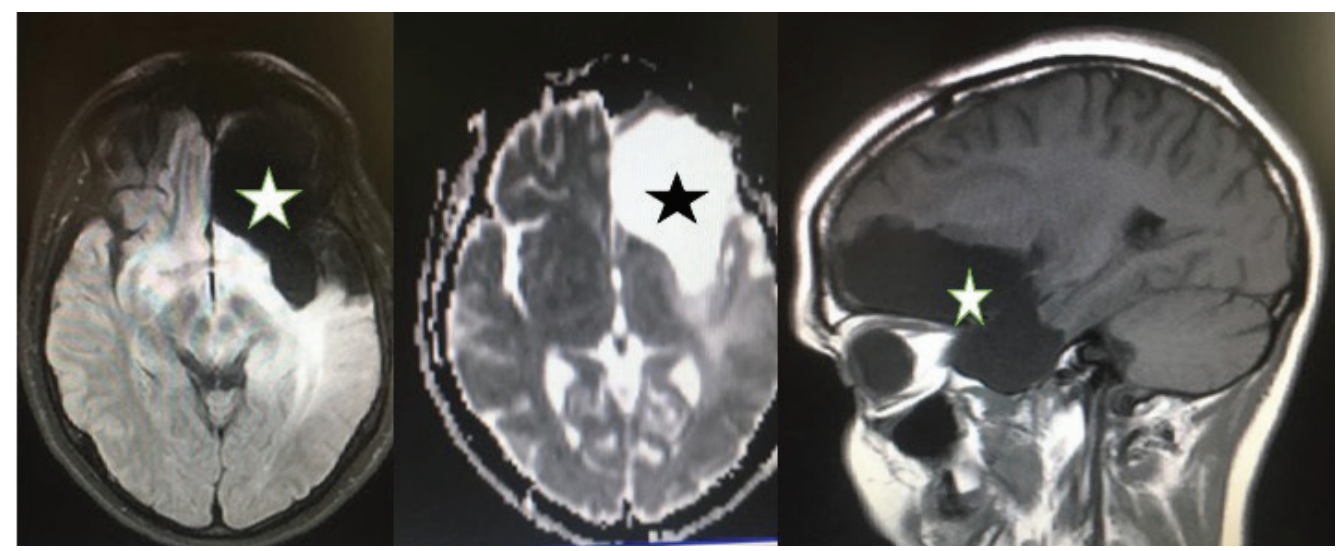

\section{Discusión}

La cirugía con monitoreo de funciones neurológicas comenzó a usarse en la década del '30, pero recién en las décadas del '70 y ' 80 se empieza a hacer de forma racional y reglada para tratar tumores cerebrales y epilepsia ${ }^{(4,5,9,31-33)}$.

En los últimos 20 años se han publicado cientos de artículos demostrando que el uso de neurofisiología intra-operatoria mejora el pronóstico funcional y aumenta el grado de resección en muchos tipos de lesiones tumorales, vasculares o epilepsia. Dentro de las técnicas de neurofisiología intra-operatoria, la cirugía con despertar tiene varios puntos favorables.

Permite personalizar el grado de resección y riesgos funcionales de cada paciente; no es un procedimiento de alto costo económico; se requiere un grupo humano entrenado pero no necesita una gran infraestructura tecnológica; su precisión para ubicar áreas funcionales es superior a cualquier otra técnica (como por ejemplo, la ubicación de áreas con reparos anatómicos o con resonancia funcional) ${ }^{(34)}$.

Pero otro lado, no todos los pacientes son candidatos a este tipo de procedimientos: individuos muy jóvenes, añosos, con claustrofobia, alteraciones siquiátricas o insuficiencia respiratoria no son buenos candidatos para estos procedimientos ${ }^{(34)}$.

\section{Etapas del procedimiento quirúrgico}

Existen varias técnicas de este tipo de cirugía, por ejemplo, Duffau y Berger (dos referentes mundiales de la cirugía tumoral con despertar operatorio) hacen el procedimiento con paciente

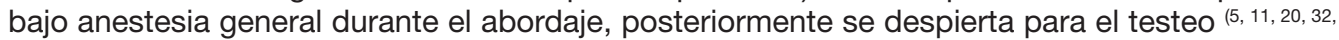
${ }^{35,36)}$. El cierre de duramadre y piel vuelven a hacerlo con el paciente dormido. Esta técnica se denomina en la literatura anglosajona como "Asleep-Awake-Asleep".

Lovo y cols en más de 200 cirugías, realizan todo el procedimiento con el paciente despierto bajo sedación al inicio ${ }^{(37)}$. Finalmente, otros autores hacen el abordaje con el paciente bajo anestesia general y tanto el testeo y la resección como el cierre, los realizan con el paciente despierto.

No está claro que haya ventajas de una técnica sobre otra.

En nuestros primeros casos se realizó la técnica aprendida con Duffau, actualmente se realiza el abordaje con anestesia general, en tanto que el testeo y resección con el paciente despierto.

\section{Indicaciones del despertar intra-operatorio}

Hay que tener claras dos cosas: no todas las lesiones se benefician de esta técnica, ni todos los pacientes pueden ser intervenidos con estas características.

Sobre el primer punto, cualquier lesión vinculada a áreas corticales o tractos de sustancia blanca en relación a funciones elocuentes (lenguaje, motricidad, visión, cognición), se podrían beneficiar de una cirugía con despertar operatorio.

Se han reportado este tipo de cirugías en metástasis, gliomas de alto y bajo grado, malformaciones arteriovenosas, angiomas cavernosos y aneurismas (2). 
Tanto las metástasis como los angiomas cavernosos, tienen en general un plano de separación entre la lesión y el cerebro. Por ello, la elección de la técnica con despertar operatorio puede hacerse para elegir el punto de entrada hacia la lesión y posteriormente seguir bajo anestesia general. Incluso si la lesión está cerca de áreas o tractos motores, el monitoreo puede hacerse con el paciente bajo anestesia general anestesiado pero sin curarizar. El estímulo del área motora en estas circunstancias provocará una contracción tónica del miembro superior (corteza de la convexidad), permitiendo ubicar el área motora primaria. Sin embargo, realizar el testeo con el paciente despierto podría tener más sensibilidad que el testeo bajo anestesia general.

Para gliomas de alto y bajo grado, el panorama es diferente. Se trata de tumores infiltrantes, sin un claro plano de separación entre el cerebro y el tumor y por lo tanto, el límite de resección no es neto. El tejido tumoral tiene una consistencia diferente al tejido cerebral normal y el cirujano puede decidir donde parar la resección en base a este cambio de consistencia. Estudios de anatomía patológica muestran que las zonas de cerebro "normal" peritumoral, están infiltradas hasta 2 centímetros más allá de donde termina el tumor macroscópicamente. Por lo tanto, a menos que se reseque la lesión con margen, no se puede asegurar de ninguna forma la resección completa y en este tipo de tumores, la recidiva es la regla ${ }^{(38)}$.

Podríamos concluir que, cualquier lesión intra-axial en relación con áreas corticales o tractos de sustancia blanca vinculados a funciones elocuentes, es candidata a realizar cirugía con despertar operatorio.

\section{Selección del paciente}

No parece haber un criterio estandarizado para seleccionar los pacientes, salvo algunas características claras. El Comité de Cirugía con Despertar Operatorio de la Sociedad Japonesa de Neurocirugía recomienda los siguientes parámetros como negativos para este tipo de cirugía: individuos de entre 15 y 65 años, portadores de tumores primitivos, metástasis o epilepsia y pone como contraindicación relativa la obesidad (39). Por la disposición de los campos operatorios, la claustrofobia es una contraindicación relativa. En nuestra serie un paciente era clasutrofóbico y por ello simulamos la posición quirúrgica y la disposición de los campos operatorios antes del procedimiento para valorar la tolerancia. Como la misma fue buena, se decidió llevar a cabo el procedimiento, que se realizó sin inconvenientes. Otras contraindicaciones (absolutas o relativas) son los trastornos siquiátricos, la falta de comprensión de cómo será el procedimiento, trastornos ventilatorios de base (EPOC, insuficiencia respiratoria).

Para los autores es crucial la percepción del cirujano y del equipo en su conjunto sobre la tolerancia del paciente. El mismo debe entender que este tipo de cirugía se realiza para beneficiarlo y no para "experimentar en su cerebro". Debe entender que se colocará un sistema de fijación que le mantendrá la cabeza fija con tres pinchos que se clavan al cráneo. Por lo tanto, si se mueve, puede lesionarse la piel.

Con nuestros criterios de selección, se lograron niveles de ansiedad intra-operatorios bajos o medios según la escala STAI (State Traite Anxiety Inventory). Un 33\% manifestó miedo a morir o tener secuelas postoperatorias. Para lograr estos niveles de colaboración, es fundamental lograr una excelente comunicación entre los integrantes del equipo, el paciente y su familia.

\section{Ampliar el margen de resección con seguridad: neurocirugía oncológica funcional}

En cirugía de gliomas es crucial lograr la resección lo más amplia posible.

El primer trabajo prospectivo comparando los grados de resección y su impacto es de Vuorinen ${ }^{(40,41)}$. En el mismo se demuestra que las resecciones amplias mejoran el pronóstico vital con respecto a la biopsia. Sin embargo es necesario separar dos escenarios: gliomas de alto y bajo grado.

\section{a) Gliomas de alto grado}

Se trata de un grupo heterogéneo de lesiones agresivas, infiltrantes y donde la recidiva es la regla (Pang). En los inicios de la neurocirugía oncológica, incluso se hicieron hemisferectomías (resección de un hemisferio cerebral) sin lograr curación ${ }^{(41,42)}$.

Al tratarse de un grupo que engloba varios tipos de tumores (astrocitomas, oligodendrogliomas y oligoastrocitomas grados 3 y 4 y glioblastomas multiformes), es difícil generalizar conductas y pronósticos. 
Varios trabajos han demostrado que a mayor grado de resección, mayor sobrevida ${ }^{(43)}$, sin embargo el grado de resección necesario para lograr un impacto significativo en la sobrevida debe ser mayor a $98 \%$ en glioblastomas para algunos autores. Con esto, se logran sobrevidas promedio de 16-18 meses con resecciones amplias.

Para lograr estos grados de resección se describen varias técnicas (disponibles en algunos centros de nuestro medio), que usan marcadores que tienen afinidad por receptores superficiales de las células tumorales y por lo tanto, permiten reconocer tejido "normal" infiltrado y decidir su remoción. Pero el límite de la resección a nivel cerebral es muy complejo de marcar si no se tiene noción de la función de ese tejido. No es posible hacer resección con margen, con seguridad de no dañar tejido neural y por lo tanto, dejar una secuela invalidante. Así y todo, tampoco se puede asegurar la resección completa y curación por más que se amplíen los márgenes de resección. Por ello, normalmente la cirugía se detiene teniendo en cuenta parámetros imagenológicos (lo que el cirujano ve en la RM preoperatoria, uso de RM intraoperatoria), quirúrgicos (lo que el cirujano identifica como tumor en el intraoperatorio usand el microscopio o 5 ALA), funcionales (resonancia funcional, neurofisiología intraoperatoria) y obviamente, se debe tener en cuenta la opinión del paciente ${ }^{(34,38)}$.

La cirugía busca: lograr una citorreducción que mejore el resultado de las otras terapias (quimioterapia, radioterapia, inmunoterapia), lograr diagnóstico anatomopatológico del grado de tumor y sus marcadores moleculares, mejorar los síntomas neurológicos deficitarios, disminuir la incidencia de crisis epilépticas y por supuesto, mejorar la sobrevida.

Por ello es importante tanto la primera cirugía como las reintervenciones. Las mismas deben hacerse con la mayor seguridad posible dado que, si impactamos en el tiempo de sobrevida, esta tiene que tener una buena calidad. Por todo esto, se debe ofrecer a los pacientes con gliomas, la cirugía más segura posible. Desde el punto de vista filosófico (pero siempre respetando la voluntad del paciente), no tiene sentido mejorar la sobrevida 4-6 meses a costo de un déficit severo que no va a permitir al paciente hacer una vida normal en los meses ganados.

En una revisión sobre los resultados de volumen de resección y aparición de nuevos déficits, Saito et al encuentran que la cirugía con despertar operatorio se asocia a niveles de resección similares o mayores que la cirugía bajo anestesia general, con una incidencia de nuevos déficits claramente menores: entre 3.4 y $7 \%$ de nuevos déficits con cirugía con despertar, contra 8.2 a $23 \%$ bajo anestesia general ${ }^{(39)}$. Nuestros resultados son absolutamente comparables a lo publicado internacionalmente en cuanto a la aparición de nuevos déficits permanentes.

Se puede aplicar la resección con margen funcional a tumores malignos que no pueden resecarse completamente o que su pronóstico vital es malo. En este caso, se puede hacer una resección basada en las expectativas del paciente.

Por ejemplo, el paciente mostrado en la figura 2 se trata de un varón portador de un glioblastoma multiforme frontal izquierdo. Se operó en una primera oportunidad con una resección muy amplia (macroscópicamente casi completa) y cerca de dos años después recidivó. La recidiva se dio por detrás de la lesión original, en relación con el área cortical del lenguaje. $A$ pesar de que el paciente tenía un trastorno del lenguaje, se realizó monitoreo intraoperatorio. Se preservo el área y vías subcorticales relacionadas con el lenguaje a pesar de no haber hecho una resección completa del tumor, ya que este fue el objetivo discutido con la familia y el paciente.

Si bien el número de gliomas de alto grado es muy bajo para sacar conclusiones, logramos resecciones totales o subtotales en 5 de 6 casos.

Por tanto, al tener muchos años de experiencia en cirugía de tumores bajo anestesia general, no es necesaria una larga curva de aprendizaje para optimizar los resultados de la cirugía con despertar.

\section{b) Gliomas de bajo grado}

Para los gliomas de bajo grado los parámetros cambian. En general los pacientes afectados son jóvenes y al tratarse de tumores de lento crecimiento, crecen infiltrando tractos de sustancia blanca y áreas corticales vinculadas con funciones elocuentes.

Al momento del diagnóstico, pueden ser voluminosos, lo que hace que su resección sea muy riesgosa desde el punto de vista funcional.

Por ello, se han planteado una gran cantidad de alternativas terapéuticas: control clínico imagenológico, biopsia, cirugía resectiva total, cirugía resectiva supratotal, biopsia seguida de radioterapia, cirugía seguida de radioterapia y/o quimioterapia ${ }^{(44)}$. 
Es difícil estandarizar una conducta, pero está claro que a mayor tamaño, más riesgos funcionales y menos posibilidad de resección completa. Igualmente, la sobrevida y la calidad de vida se ven afectadas.

Frente a estos problema, Duffau plantea nuevos paradigmas en la cirugía de gliomas de bajo grado (GBG) la resección con límites funcionales y la resección supra-máxima (15).

Esto implica cambios en la concepción de la cirugía de estos tumores. Por ejemplo, dado que los GBG son tumores de lento crecimiento que afectan a personas jóvenes y que en general son asintomáticos o muy poco sintomáticos, una conducta puede ser el "ver y controlar". Lo que ocurre es que estos tumores continúan su crecimiento y poco a poco van invadiendo tractos subcorticales de sustancia blanca. Por esto, mientras más tiempo pase, más grande será el tumor, mas áreas y tractos de sustancia blanca se verán comprometidos y por tanto, más compleja la resección.

Duffau adaptó técnicas usadas en cirugía de epilepsia a la cirugía tumoral y es en este momento, uno de los cirujanos con más experiencia en el mundo en cirugía de GBG con despertar intraoperatorio.

¿Qué ventajas tiene? Si el paciente se opera despierto, el cirujano puede testear función, y si el tejido que parece normal no genera ningún déficit, puede resecarse. Esto mejora el pronóstico vital a largo plazo al permitir resecciones más amplias y la única forma segura de hacerla es con el paciente despierto. Esta cirugía, cuando excede los límites de tejido tumoral visible pero respeta límites funcionales se denomina "resección supra-máxima". Con esta técnica Duffau ha duplicado el tiempo de sobre vida de un grupo de pacientes, pero obviamente no es aplicable a todos los portadores de tumores cerebrales y ni siquiera es aplicable a todos los individuos con GBG. En nuestra opinión debería ser la opción a elegir cuando sea posible según las características del paciente y la lesión.

Vemos entonces que la cirugía con margen funcional permite dos opciones: resecar el tumor con margen libre (deteniendo la resección donde se ubica una función importante) o detener la resección dejando tumor para conservar una función. Incluso en casos seleccionados, donde se ven afectados tractos que cuentan con "circuitos de seguridad", es aceptable un déficit mínimo, apelando a la plasticidad cortical. De hecho en nuestros pacientes tuvimos 4 déficits transitorios que revirtieron en semanas.

Nuevamente, si bien el número de pacientes es bajo y a pesar de que algunos tenían tumores muy extensos, se logró resección subtotal o supra-máxima en 5/6.

\section{Complicaciones}

Muchas veces se repite que en nuestro país tenemos una población pequeña y por lo tanto, no se pueden lograr resultados similares a grandes centros de nuestra región en cuanto a sobrevida, grados de resección y complicaciones.

En cuanto a la aparición de nuevos déficits, se reportan cifras de entre 0 y $9 \%{ }^{(45-47)}$. Esto depende de la experiencia del cirujano, la extensión de la lesión, la colaboración del paciente, la presencia de déficits previos, etc. En cuanto a la aparición de nuevos déficits que no revierten, nuestros números son aceptables y comparables a centros de referencia a pesar de que el número de pacientes no es alto. Incluso las cifras que tenemos son comparables a algunas series presentadas por el propio Duffau. Luego de completar esta serie duplicamos el número de cirugías con presencia de déficits permanentes comparable. De todas formas, sería deseable continuar bajando la incidencia de déficits transitorios que idealmente deberían ser del entorno del $1 \%$, cifra lograda por centros altamente especializados.

El estímulo intra-operatorio de la corteza cerebral puede producir convulsiones, lo que altera el desarrollo del procedimiento y eventualmente puede hacer que el monitoreo no sea fiable. La presencia de crisis intra-operatorias están reportadas entre el $5 \%$ y el $9.5 \%$ de los casos. Nuestra incidencia es mayor a lo reportado, aunque no tuvo impacto en la cirugía, más que prolongar el tiempo de la misma. Esto es debido a que para lograr una colaboración confiable se debe tener un paciente colaborando y si las crisis alteran la conciencia, se debe esperar a que esta se recobre completamente.

Luego del despertar, es importante que el paciente colabore en los testeos de forma activa. Si no es capaz de hacerlo o su capacidad de tolerancia es baja, no se terminará beneficiando del procedimiento por lo que el mismo se debería interrumpir. Para Sarang (48) la incidencia de despertar excitado o sin capacidad de colaboración puede llegar hasta el 19\%, esta cifra se puede reducir con una correcta selección de los pacientes. 
En nuestro grupo, un 9\% de los pacientes no pudo lograr un nivel de colaboración adecuado. Esta cifra también está dentro de lo reportado, pero es deseable que sea más baja aún, lo que se logra puliendo el proceso de selección previo.

Para terminar, la incidencia de infecciones también está dentro de lo reportado $(4.5 \%)$ aunque también pensamos que esta cifra debe ser más baja.

La mortalidad reportada para este tipo de procedimientos esta entre 0 y $2 \%$. No hubo ninguna muerte en nuestros pacientes, ni en el postoperatorio inmediato ni a 30 días.

Vemos entonces que para todos los tipos de complicaciones analizadas, nuestros resultados son comparables a lo publicado en la literatura.

\section{Conclusiones}

Se presenta una serie de 20 pacientes operados con despertar operatorio en los que se hicieron 22 cirugías.

Los grados de resección logrados, así como la presencia de complicaciones, está en concordancia con lo reportado en la literatura mundial.

Los autores consideran que la cirugía con despertar operatorio es segura, confiable y sus resultados son reproducibles luego de una muy corta curva de aprendizaje para cirujanos que tienen experiencia previa en resección de tumores con técnicas convencionales.

\section{Agradecimientos}

Al Prof. Hugues Duffau (Hospital Universitario Henri Mondor, Montpellier, Francia) por sus enseñanzas y aportes. El Prof. Duffau recibió en su servicio a 4 autores del presente artículo (FM, RM, FS, JMM) y es consultante de nuestro equipo para casos complejos.

\section{Bibliografía}

1- Schott GD. Penfield's homunculus: a note on cerebral cartography. J Neurol Neurosurg Psychiatry 1993;56:329-33.

2- Abdulrauf SI. Awake craniotomies for aneurysms, arteriovenous malformations, skull base tumors, high flow bypass, and brain stem lesions. J Craniovertebr Junction Spine. 2015;6:8-9.

3- Akay A, Islekel S. Awake craniotomy procedure: Its effects on neurological morbidity and recommendations. Turk Neurosurg 2018;28:186-192.

4- Martinez F. Moragues R. Cirugía tumoral con despertar intraperatorio: es posible en Uruguay? Opción Medica 2016;61:4-8.

5- Zhang JJY, Lee KS, Voisin MR, Hervey-Jumper SL, Berger MS and Zadeh G. Awake craniotomy for resection of supratentorial glioblastoma: a systematic review and meta-analysis. Neurooncol Adv 2020;2:vdaa111.

6- Martínez F, Dominguez-Perez M, Cuadros M, Moragues R, Segura Fernandez M, Casales N, et al. Tumores de los nervios periféricos: estudio retrospectivo de 66 casos. Neurocirugía (Astur) 2020;31(3):105-111.

7- Martínez F, Pinazzo S, Armand Ugon G, Diamant M, Gonzalez F. Abordaje multidisciplinario a tumores del plexo lumbar. A propósito de dos casos. Rev Latinoamer Neurocir (FLANC). 2016;21(1):8p

8- Martínez F, Salle F, Moragues R, Bertullo G. Utilidad de la estimulación intra- operatoria en cirugía de procesos expansivos intracraneanos bajo anestesia general. Rev Uru Med Int 2017;1:58-63.

9- Moragues R, Martinez F. Tumores intracraneanos. En: Braga P, Martínez F (Eds): Manual Básico de Neurología y Neurocirugía para estudiantes y médicos generales. Montevideo: Oficina del Libro, 2019:47-52.

10- Martinez F. Technical Note: Spinal-Accessory to Suprascapular nerve transfer by posterior approach. Austin Neurosurg Open Acces. 2017;4(1):1058.

11- Duffau H. Surgery for diffuse low grade gliomas (DLGG). Oncological considerations.. In: Duffau H (Ed): Difuse low grade gliomas in adults. London: Springer, 2013. p.359-374. 
12- Duffau H. Awake mapping is not an additional surgical technique but an alternative philosophy in the management of low-grade glioma patients. Neurosurg Rev 2018;41:689-691.

13- Yordanova YN, Moritz-Gaser S, Duffau H. Awake surgery for WHO Grade II gliomas within "noneloquent" areas in the left dominant hemisphere: Toward a "supratotal" resection. Clinical article. J Neurosurg 2011;115:232-239.

14- De Witt Hamer PC, Moritz-Gaser S, Gatignol P, Duffau H. Is the human left middle longitudinal fascicle essential for language? A brain electrostimulation Study. Human Brain Mapping 2011;32:962-973.

15- Duffau H,Tailandier L. New concepts in the management of diffuse low-grade glioma: Proposal of a multistage and individualized therapeutic approach. Neuro Oncol 2015;17(3):332-342.

16- Martínez F, Salle F, Moragues R, Bertullo G. Utilidad de la estimulación intra- operatoria en cirugía de procesos expansivos intracraneanos bajo anestesia general. Rev Urug Med Interna. 2017:58-63.

17- Duffau H, Tailandier L. New indivuadilzed strategies in DLGG. In: Duffau H (Ed): Difuse low grade gliopmas in adults. London:Springer, 2013. p. 435-446.

18- Duffau H, Denvil D, Sichez N, et al. Functional recovery after surgical resection of low grade gliomas in eloquent brain: hypothesis of brain compensation. J Neurol Neurosurg Psychiatry 2003;74:901-907.

19- Byrne RW, Sanai N, Landeiro JA and Duffau H. Introduction: Advances in intraoperative brain mapping. Neurosurg Focus 2018;45.

20- De Benedictis A, Moritz-Gasser S, Duffau H. Awake mapping optimizes the extent of resection for lowgrade gliomas in eloquent areas. Neurosurgery 2010;66:1074-1084.

21- Duffau H. Paradoxes of evidence-based medicine in lower-grade glioma: To treat the tumor or the patient? Neurology 2018;91:657-662.

22- Ferracci FX, Duffau H. Improving surgical outcome for gliomas with intraoperative mapping. Expert Rev Neurother 2018;18:333-341.

23- Moritz-Gasser S. Language and other cognitive evaluations. In: Duffau H (Ed): Difuse low grade gliomas in adults. Springer, London 2013:279-300.

24- Mandonnet E, Duffau H. An attempt to conceptualize the individual onco-functional balance: Why a standardized treatment is an illusion for diffuse low-grade glioma patients. Crit Rev Oncol Hematol 2018;122:83-91.

25- Duffau H. Early and maximal personalized surgical resection improves survival and quality of life in lowgrade gliomas patients. Neurol India 2020;68:813-814.

26- Duffau H, Arthuis F, Bitar A, Sichez JP, Van Effenterre R, Capelle L. Contribution of intraoperative electrical stimulations in surgery of low grade gliomas: a comparative study between two series without (1985-96) and with (1996-2003) functional mapping in the same institution. J Neurol Neurosurg Psychiatry 2005;76(6):845-851.

27- Sainz L, Ratto F, Munua F, et al. Experiencia emocional percibida por pacientes sometidos a craneotomía con despertar intraoperatorio. Rev Urug Med Interna 2019;1:32-39

28- Guzmán J. LMA Supreme. Rev Chil Anest, 2009; 38: 114-118 2009.

29- Fay MA, Margulis L. Test de denominación de Boston: rendimiento de jóvenes adultos de la ciudad autonoma de Buenos Aires. An Invest Fac Psic 2018;25:343-351.

30- Martinez-Cuitiño M. Pirámides y palmeras o pirámides y faraones?: Adaptación y validación de un test de asociación semántica al español rioplatense. Interdisciplinaria Rev Psicol Ciencias Afines 27(2):247-260.

31- Lovo M, Ahues E, Martinez Cortez R, Milla R, Cruz C. Seguridad y tolerabilidad de la craneotomía vigíl para tumores cerebrales y otras lesiones supratentoriales. Rev Argent Neuroc 2018;32:258-264.

32- Hervey-Jumper SL, Berger M. Technical nuances of awake brain tumor surgery and the role of maximum safe resection. J Neurosurg Sci 2015;59(4):351-360.

33- Duffau $\mathrm{H}$. Is non-awake surgery for supratentorial adult low-grade glioma treatment still feasible? Neurosurg Rev 2018;41:133-139.

34- Huart N, Biaffore F, Martínez F. Que es la resonancia magnética funcional de cerebro y cuales son sus aplicaciones? Opción Médica 2019;78:38-40. 
35- Yordanova YN, Cochereau J, Duffau H, Herbet G. Combining resting state functional MRI with intraoperative cortical stimulation to map the mentalizing network. Neuroimage 2019;186:628-636.

36- Duffau $\mathrm{H}$. Awake mapping is not an additional surgical technique but an alternative philosophy in the management of low-grade glioma patients. Neurosurg Rev 2018;41:689-691.

37- Berger MS, Dobbins J, Keles GE. The effect of extent of resection on recurrence in patients with lowgrade cere- bral hemisphere gliomas. Cancer 1994;74(6):1784-1791.

38- Duffau H. Surgery for malignant brain gliomas: Fluorescence-guided resection or functional-based resection? Front Surg 2019;6:21.

39- Saito T, Maruyama T, Tamura M, Nitta M, OkadaY. Intraoperative functional mapping and monitoring during glioma surgery. Neurol Med Chir (Tokyo) 2015;55:1-13.

40- Vuorinen V, Färkkilä M, Jääskeläinen J. Debulking or biopsy of malignant glioma in elderly people - a randomised study. Acta Neurochir (Wien) 2003;145(1):5-10.

41- Lhermitte J. L'ablation complète de l'hemisphère droit dans les cas des tumeur cérébrale localisée compliquée d'hémiplégie. La décérébration supra-thalamique unilatérale chez l'homme. Encephale 1928;23:314-323.

42- Martínez F, Soria V, Sgarbi N, Laza S, Prinzo H. Bases anatómicas de la hemisferotomía periinsular. Rev Méd Urug 2004;20(3):208-214.

43- Pang B, Lee CK, Chu KJ, Ng WH. The Role of Surgery in High-grade Glioma - Is Surgical Resection Justified? A Review of the Current Knowledge. Ann Acad Med Singapore 2007;36:358-363.

44- Lhiff MN KGM. The ethical dilemma in the surgical management of low grade gliomas according to the variable availability of resources and surgeon experience. Asian J Neurosurg 2020;15(2):266-271.

45- Wilson SM, Lam D, Babiak MC, et al. Transient aphasias after left hemisphere resective surgery. J Neurosurg 2015;123:581-93.

46- Bu LH, Zhang J, Lu JF and Wu JS. Glioma surgery with awake language mapping versus generalized anesthesia: a systematic review. Neurosurg Rev 2020.

47- Sanai N, Berger M. Operative techniques for gliomas and the value of extent of resection. Neurotherapeutics 2009;6:478-486.

48- Sarang A, Dera J. Anaesthesia for awake craniotomy. Evolution of a technique that facilitates awake neurological testing. Brit J Anaesth 2003;90(2):161-165.

\section{Aporte de cada autor al trabajo}

Fernando Martínez: Concepción y diseño, recolección de datos, interpretación de datos, revisión crítica del trabajo.

Rodrigo Moragues: Concepción y diseño, interpretación de datos, revisión crítica del trabajo. Gonzalo Bertullo: Recolección de datos, interpretación de datos, revisión crítica del trabajo. Juan Marcos Mira: Recolección de datos, interpretación de datos, revisión crítica del trabajo.

Federico Salle: Recolección de datos, interpretación de datos, interpretación de datos, revisión crítica del trabajo.

Gastón Duarte: Redacción del trabajo, revisión crítica del trabajo.

Ronnie Henderson: Redacción del trabajo.

Fabricio Sachet: Redacción del trabajo, revisión crítica del trabajo.

Federico Preve: Recolección de datos, interpretación de datos, revisión crítica del trabajo.

Andrés Lescano: Recolección de datos, interpretación de datos.

Gustavo Grunberg: Redacción del trabajo, revisión crítica del trabajo.

Lorena Tambasco: Redacción del trabajo.

Paola Alcarraz: Redacción del trabajo.

Elena Vignoli: Redacción del trabajo, revisión crítica del trabajo. 